\title{
Clinical and genetic characterization of a Chanarin Dorfman Syndrome patient born to diseased parents
}

\author{
Murat Durdu', Sara Missaglia ${ }^{2,3}$, Laura Moro ${ }^{4}$ and Daniela Tavian ${ }^{2,3^{*}}$ (B)
}

\begin{abstract}
Background: Chanarin Dorfman Syndrome (CDS) is a rare autosomal recessive disorder characterized by ichthyosiform non-bullous erythroderma and variable involvement of the liver and the neuromuscular system. In CDS patients, the accumulation of neutral lipids inside cytoplasmic lipid droplets has been demonstrated in different tissues. To date, ninety families with this disease have been described worldwide; most of them are from Mediterranean countries.

Case presentation: In this report, we describe a consanguineous Turkish family with typical features of CDS. The parents are first cousins and are both diseased. At the age of eight, their child presented CDS with non-bullous congenital ichthyosiform erythroderma, hepatosteatosis, hepatomegaly and ectropion. Electromyographic examination is compatible with myopathy. A five-year-old cousin of the child is also affected by CDS. She was born to non-affected consanguineous parents. Mutation analysis of the ABHD5 gene revealed the previously reported mutation, N209X, which is the most frequent in Turkish patients. Lipid vacuoles, also known as Jordan's anomaly, are detectable in their leucocytes.

Conclusions: To the best of our knowledge, this is the first report of a CDS family in which both parents and their child are affected by CDS. To date, the child does not present a more severe clinical phenotype compared with those of his relatives or other CDS patients of the same age. These findings suggest that high levels of triacylglycerol accumulation, that may be supposed to be present in high amount inside the ooplasm, did not affect embryo development and foetal growth.
\end{abstract}

Keywords: Chanarin-Dorfman Syndrome, Ichthyosis, Lipid disorder, Liver involvement, Myopathy

\section{Background}

Neutral lipid storage disease with ichthyosis, which is also known as Chanarin Dorfman Syndrome (CDS; MIM 275630), is a rare autosomal recessive disease characterized by the intracellular accumulation of triacylglycerol (TG) in numerous tissues $[1,2]$. The clinical phenotype involves multiple organs and systems, including skin, liver, skeletal muscle, eyes, ears, and the central nervous system. However, in this syndrome, the degree of systemic involvement is quite variable $[2-5]$. ABHD5 ( $\alpha / \beta$ hydrolase domain 5$)$, a cofactor for adipose triglyceride lipase (ATGL), has been

\footnotetext{
* Correspondence: daniela.tavian@unicatt.it

²Laboratory of Cellular Biochemistry and Molecular Biology-CRIBENS, Catholic University of the Sacred Heart, pz Buonarroti 30, 20145 Milan, Italy ${ }^{3}$ Department of Psychology, Catholic University of the Sacred Heart, Largo Gemelli 1, 20123 Milan, Italy

Full list of author information is available at the end of the article
}

identified as a causative gene of CDS [6]. Indeed, different ABHD5 mutations determine the partial or total loss of ATGL activation, leading to the accumulation of TG inside lipid droplets [2-5]. Lipid droplets are highly dynamic and ubiquitous cellular organelles. These droplets are a fundamental component of lipid homeostasis, i.e., a universal feature of eukaryotic cells that can ensure a rapidly mobilized lipid source for numerous biochemical processes [7]. Neutral lipids also accumulate in oocytes and blastomeres as lipid droplets, providing energy for mammalian embryo preimplantation, proper growth and development [8].

In the peripheral leukocytes of CDS patients, neutral lipids inside lipid droplets are easily detectable with standard or specific stains, and they are known as Jordan's anomalies, the most common laboratory findings of the disease [9].

(c) The Author(s). 2018 Open Access This article is distributed under the terms of the Creative Commons Attribution 4.0 International License (http://creativecommons.org/licenses/by/4.0/), which permits unrestricted use, distribution, and 
One hundred and twenty-eight CDS patients have been reported worldwide (Additional file 1: Table S1). For 85 of these patients, clinical diagnosis has been confirmed by $A B H D 5$ mutation analysis. The highest number of CDS patients (27 cases) has been described in Turkey, mainly due to the high incidence of consanguineous marriages [10]. Nevertheless, the clinical and genetic description of families in which both parents and children are affected by CDS has never been reported before.

\section{Case presentation}

Here, we present a CDS Turkish family with four affected members (Fig. 1a, III-1, III-2, IV-1, IV-2). The child (IV-1) was an 8-year-old male born at 38 weeks of gestation and weighing $3400 \mathrm{~g}$. He presented lamellar ichthyosis at birth.
At first examination (at the age of 5), he had lagophthalmos and ectropion. Ophthalmologists did not record lagophthalmos in subsequent examinations, so the condition was considered a compliance problem. He had elevated transaminase (ALT: $75 \mathrm{U} / \mathrm{L}$; normal 5-35 U/L; GGT $31 \mathrm{U} / \mathrm{L}$; normal 5-17 U/L), creatine phosphokinase (CK: $1677 \mathrm{U} / \mathrm{L}$; normal 22-200 U/L), triglyceride (460 mg/dl; normal $35-130 \mathrm{mg} / \mathrm{dl}$ ), and total cholesterol levels (135 mg/dl; normal 110-200 mg/dl) (Table 1). Systemic examination revealed $2 \mathrm{~cm}$ hepatomegaly. Abdominal ultrasonography was compatible with hepatosteatosis and hepatomegaly. Electromyographic examination revealed signs of myopathy.

His parents (both 44 years old) were paternal cousins. Both presented lamellar ichthyosis, ectropion, hypertriglyceridemia,

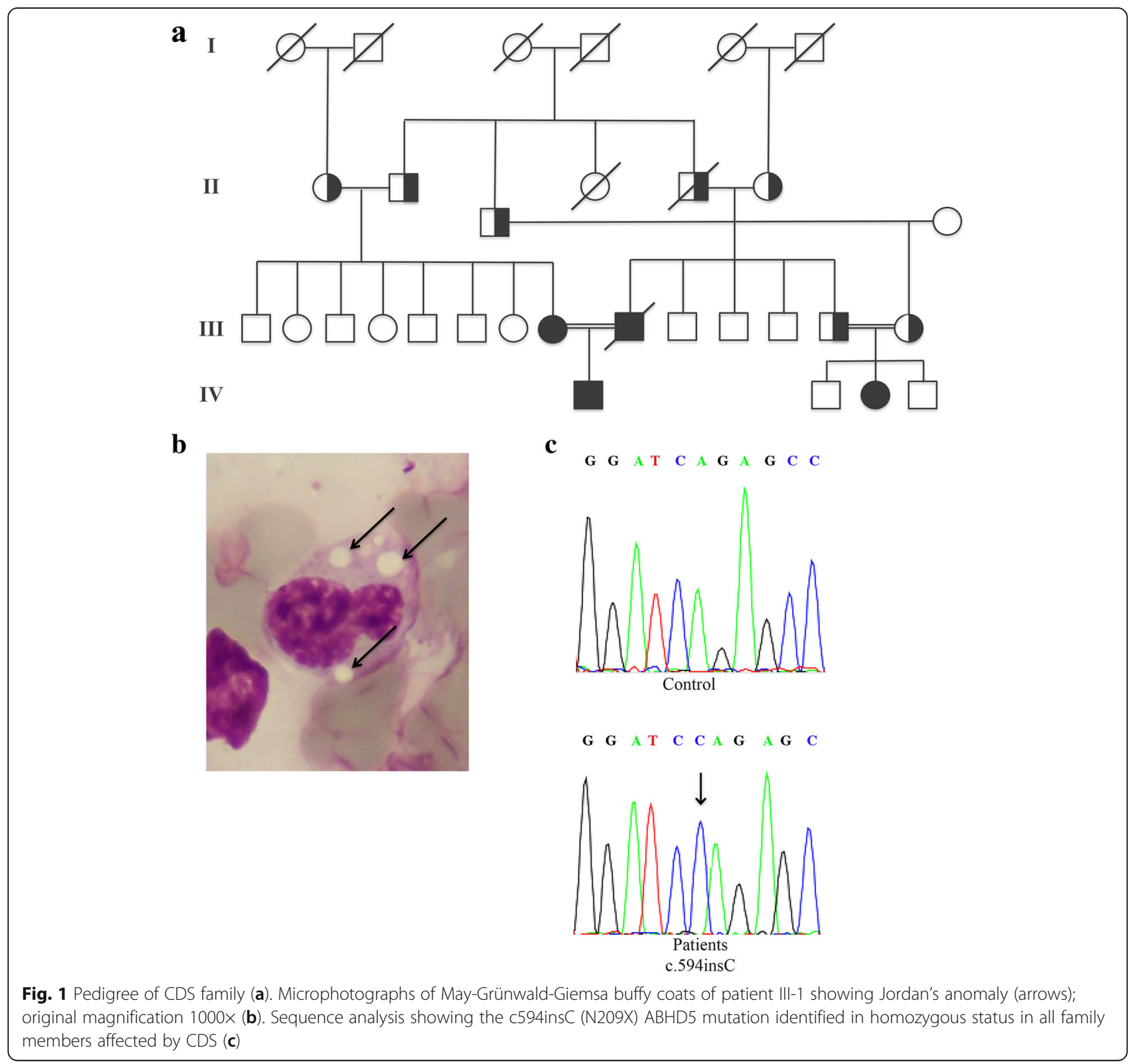


Table 1 Clinical and laboratory features of patients with Chanarin-Dorfman syndrome

\begin{tabular}{|c|c|c|c|c|}
\hline Clinical feature & Child & Father & Mother & Cousin \\
\hline Ichthyosis & + & + & + & + \\
\hline Leukocyte vacuoles & + & + & + & + \\
\hline Creatinine phosphokinase levels (U/L) & 1677 & 290 & 219 & 1145 \\
\hline Electromyographic examination & Myopathy & Normal & Normal & Myopathy \\
\hline Triglyceride level (mg/dL) & 460 & 115 & 74 & 74 \\
\hline Total cholesterol level (mg/dL) & 135 & 151 & 115 & 140 \\
\hline Cataracts & - & - & - & - \\
\hline Ectropion & + & + & + & - \\
\hline Lagophthalmos & + & - & - & - \\
\hline Strabism & - & - & - & - \\
\hline Myopia & - & + & - & - \\
\hline Hearing loss & - & - & - & - \\
\hline Mental retardation & - & - & - & - \\
\hline Hepatomegaly & $+(2 \mathrm{~cm})$ & + & + & $+(2 \mathrm{~cm})$ \\
\hline Hepatosteatosis & + & + & + & + \\
\hline Microcephaly & - & - & - & - \\
\hline Intestinal involvement & - & - & - & - \\
\hline
\end{tabular}

hepatomegaly and hepatosteatosis (Table 1). In both parents and the child, dermatological examination revealed widespread ichthyosis on the facial region, trunk, extensor and flexural regions and scalp. The individual scales over the trunk were white, fine, translucent and semi-adherent, whereas those on the limbs and face were grey-brown, larger in size, polygonal and adherent (Fig. 2). No bullous lesions or erosions were noted. The involvement of palms and soles, dental anomaly and nail dystrophy was absent.

In the 5-year-old girl (IV-2), ichthyosis was also present in flexural regions (Fig. 2). Corrugated appearance and hyperpigmented scales were detected on foot, back, knee, and elbow. Laboratory investigation revealed increased ALT (86 U/L) (alanine transaminase), AST (aspartate transaminase) (88 U/L) and CK (1145 U/L) levels (Table 1). Electromyographic examination revealed myopathy. Abdominal ultrasonography revealed hepatosteatosis causing hepatomegaly. Ophthalmological and audiological examinations were normal.

A peripheral blood smear stained with May-GrünwaldGiemsa revealed lipid vacuoles (Jordan's anomaly) in leucocytes from all patients (Fig. 1b). After obtaining informed consent and in accordance with the Declaration of Helsinki principles, the coding regions of the ABHD5 gene were sequenced, and a homozygous N209X mutation was identified (Fig. 1c). For molecular analysis, oligonucleotides were selected to amplify and sequence the seven exons of $A B H D 5$, their intron/exon boundaries, and the candidate promoter regions. The primer sequences and the amplification conditions were previously reported [2]. A few days after molecular diagnosis, the father died of a heart attack at the age of 44 . He had no prior cardiac history. However, PNPLA2 gene analysis was performed, excluding disease-causing mutations.

The CARE guidelines were followed in this case.

\section{Discussion and conclusions}

The N209X mutation identified in our CDS family is the most common in the Turkish CDS population. Nur et al. compared clinical findings between patients carrying this mutation with other ABHD5 variations and noted no significant differences [10]. In CDS patients, skin involvement is prevalent and a consistently observed clinical feature, consisting of a non-bullous congenital ichthyosiform erythroderma. Sano et al. demonstrated that the severity of ichthyosis positively correlates with TG level in the scales from patients [11]. In our affected subjects, a widespread lamellar ichthyosis detected since birth revealed a homogeneous CDS phenotype. Notably, skin involvement did not appear more severe in the child; however, it is possible to hypothesize increased accumulation of TG in ectodermal cells during embryonic development. Liver abnormalities can occur in greater than $80 \%$ of patients, ranging from hepatomegaly or liver steatosis to cirrhosis, and can be observed in young children, as occurred in this family $[4,6]$. Neurological impairment was not detected in our patients; however, intellectual disability has been reported in $20 \%$ of CDS subjects and in approximately $40 \%$ of those carrying the N209X mutation [10-12]. 

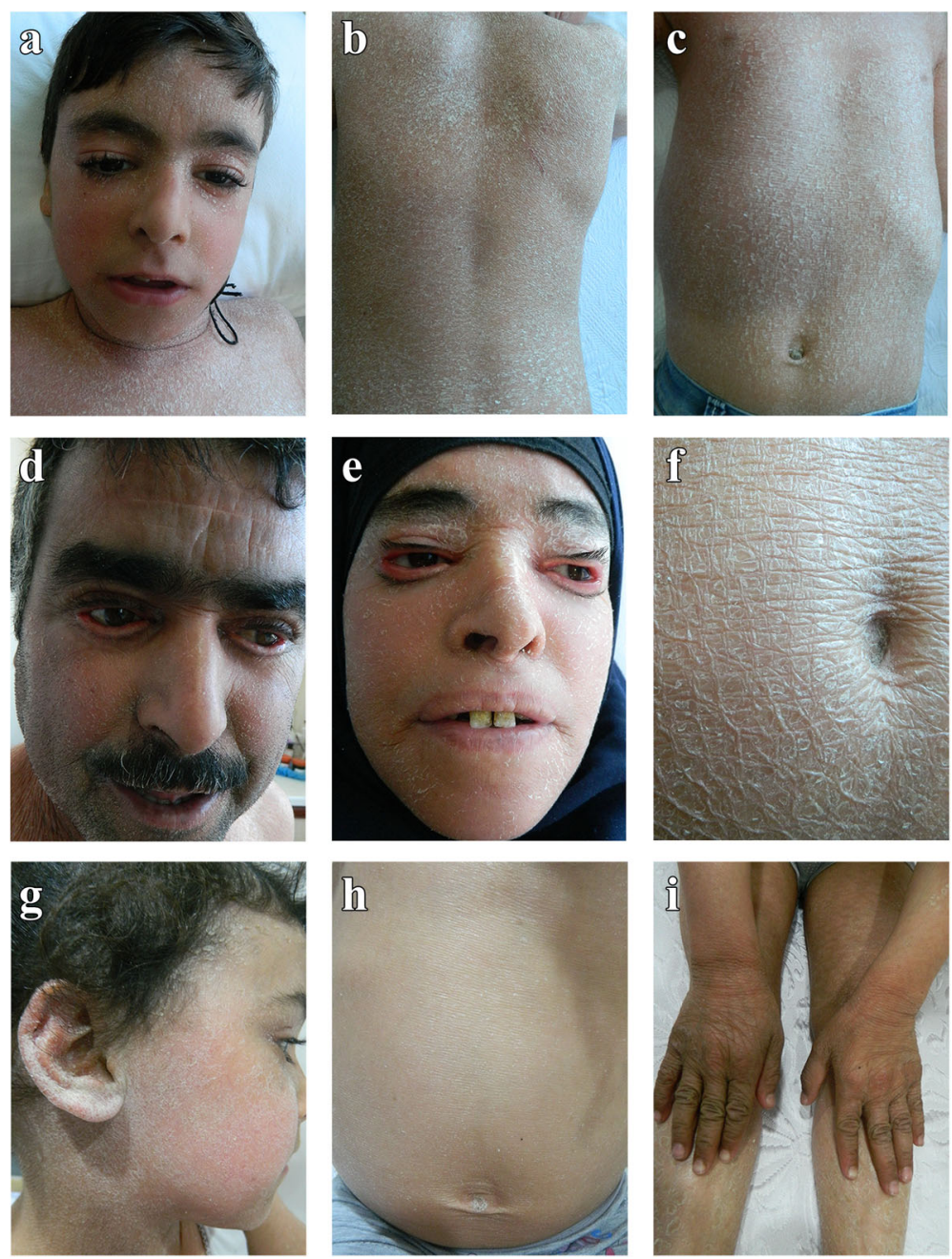

Fig. 2 Dermatological characterization of CDS patients. Lamellar ichthyosis affecting facial region $(\mathbf{a}, \mathbf{d}, \mathbf{e}, \mathbf{g})$, trunk $(\mathbf{b}, \mathbf{c}, \mathbf{f}, \mathbf{h})$, and extremities (i) of child (patient IV-1; $\mathbf{a}, \mathbf{b}, \mathbf{c}$ ), father (patient III-2; d), mother (patient III-1; e, f) and cousin (patient IV-2; $\mathbf{g}, \mathbf{h}$, i). Prominent eyelid ectropion in father (d) and mother (e)

Sensorineural hearing loss was not present in our family; however, this condition occurs in $30 \%$ of CDS patients. Muscle involvement was evident only in the two children. Clinically, the children did not exhibit significant muscle weakness, fatigue or exercise intolerance. However, an electromyographic examination (EMG) was performed because of an elevation in CK levels and revealed a myopathic pattern. In CDS, muscle abnormalities have been detected in $40 \%$ of subjects. Myopathy typically begins in the thirties, but it has also been described in very young children $[2,13]$.

In conclusion, we describe a family in which all members are affected by CDS. When the child was born, his mother was 36 years old. She did not indicate any infertility problems or history of abortion. This paper describes the unique reported case of natural conception and pregnancy between two CDS patients. No particular pregnancy complications were observed. Therefore, the storage of neutral lipids inside lipid droplets did not dramatically increase during oocyte maturation. Indeed, embryo development and foetal growth were not affected. To date, the child does not present new symptoms or an exacerbation of the clinical phenotype even with regard to dermatologic manifestations compared with the symptoms and phenotypes of his relatives or other CDS patients. Instead, a relevant aspect for these three patients seems to be represented by the psychogenic stress due to isolation from society, colleagues, school friends and relatives. Finally, after molecular diagnosis, the two children immediately started a special diet, poor in fatty acids with medium chain triglycerides (MCT), as hepatic and dermatologic improvement has been reported in different cases consuming such a diet. 


\section{Additional file}

Additional file 1: Table S1. Summary of CDS patients reported in the literature. (DOCX $30 \mathrm{~kb}$ )

\section{Abbreviations}

ABHD5: $\alpha / \beta$-hydrolase domain-containing protein 5; ALT: Alanine aminotransferase test; AST: Aspartate aminotransferase test; ATGL: Adipose triglyceride lipase; CDS: Chanarin-Dorfman syndrome; CK: Creatine kinase; GGT: Gamma glutamyl transpeptidase; LD: Lipid droplet; MCT: Medium chain triglycerides; NCIE: Non-bullous congenital ichthyosiform erythroderma; NLSDI: Neutral lipid storage disease with ichthyosis; TG: Triacylglicerol

\section{Acknowledgements}

The authors are grateful to the patients for their kind cooperation.

\section{Funding}

Università Cattolica del Sacro Cuore (Milan, Italy) sustained the publication of this article.

\section{Availability of data and materials}

Data obtained during this study are included in the article and its additional files.

\section{Authors' contributions}

MD performed the clinical characterization of patients and he is taking care of them; SM performed the molecular analysis and critically revised the manuscript; LM supervised the study and critically revised the manuscript; DT conceived the study, supervised it and wrote the manuscript. All authors read and approved the final manuscript.

\section{Ethics approval and consent to participate}

Informed consent for genetic analysis was obtained from the study participants. This manuscript reports the description of a family who presented with CDS clinical symptoms. No experiments were performed and no hypothesis were tested for this study. This case report does not constitute systematic research, therefore no ethics approval was necessary.

\section{Consent for publication}

Written informed consent was obtained from adult patients and the guardians of two young patients for publication of this Case report and any accompanying images. A copy of the written consent is available for review by the Editor of this journal.

\section{Competing interests}

The authors declare that they have no competing interests.

\section{Publisher's Note}

Springer Nature remains neutral with regard to jurisdictional claims in published maps and institutional affiliations.

\section{Author details}

${ }^{1}$ Baskent University Faculty of Medicine, Department of Dermatology, Adana Hospital, Adana, Turkey. ${ }^{2}$ Laboratory of Cellular Biochemistry and Molecular Biology-CRIBENS, Catholic University of the Sacred Heart, pz Buonarroti 30, 20145 Milan, Italy. ${ }^{3}$ Department of Psychology, Catholic University of the Sacred Heart, Largo Gemelli 1, 20123 Milan, Italy. ${ }^{4}$ Department of Pharmaceutical Sciences, University of Piemonte Orientale, Lgo Donegani 2, 28100 Novara, Italy.

Received: 19 February 2018 Accepted: 18 May 2018

Published online: 29 May 2018

\section{References}

1. Schweiger M, Lass A, Zimmermann R, Eichmann TO, Zechner R. Neutral lipid storage disease: genetic disorders caused by mutations in adipose triglyceride lipase/PNPLA2 or CGI-58/ABHD5. Am J Physiol Endocrinol Metab. 2009;297: 289-96.

2. Redaelli C, Coleman RA, Moro L, Dacou-Voutetakis C, Elsayed SM, Prati D, Colli A, Mela D, Colombo R, Tavian D. Clinical and genetic characterization of chanarin-dorfman syndrome patients: first report of large deletions in the ABHD5 gene. Orphanet J Rare Dis. 2010:5:33.

3. Bruno C, Bertini E, Di Rocco M, Cassandrini D, Ruffa G, De Toni T, et al. Clinical and genetic characterization of Chanarin-Dorfman syndrome. Biochem Biophys Res Com. 2008;369:1125-8.

4. Missaglia S, Valadared ER, Moro L, Faguntes ED, Quintão Roque R, Giardina $B$, et al. Early onset of Chanarin-Dorfman syndrome with severe liver involvement in a patient with a complex rearrangement of ABHD5 promoter. BMC Med Genet. 2014;15:32.

5. Takeichi T, Sugiura K, Tso S, Simpson MA, McGrath JA, Akiyama M. Bi-allelic nonsense mutations in ABHD5 underlie a mild phenotype of DorfmanChanarin syndrome. J Dermatol Sci. 2016;81:134-6.

6. Lefevre C, Jobard F, Caux F, Bouadjar B, Karaduman A, Heilig R, et al. Mutations in CGl-58, the gene encoding a new protein of the esterase/ lipase/thioesterase subfamily, in Chanarin-Dorfman syndrome. Am J Hum Genet. 2001;69:1002-12.

7. Welte MA. Expanding roles for lipid droplets. Curr Biol. 2015; https://doi.org/ 10.1016/j.cub.2015.04.004.

8. Prates EG, Nunes JT, Pereira RM. A role of lipid metabolism during cumulusoocyte complex maturation: impact of lipid modulators to improve embryo production. Mediat Inflamm. 2014; https://doi.org/10.1155/2014/692067.

9. Tavian D, Colombo R. Improved cytochemical method for detecting Jordans' bodies in neutral-lipid storage diseases. J Clin Pathol. 2007;60:956-8.

10. Nur BG, Gencpinar P, Yuzbasioglu A, Emre SD, Mihci E. Chanarin Dorfman syndrome: genotype-phenotype correlation. Eur J Med Genet. 2015;58:238-42.

11. Ujihara M, Nakajima K, Yamamoto M, Teraishi M, Uchida Y, Akiyama M, et al. Epidermal triglyceride levels are correlated with severity of ichthyosis in Dorfman-Chanarin syndrome. J Dermatol Sci. 2010;57:102-7.

12. Pennisi EM, Arca M, Bertini E, Bruno C, Cassandrini D. D'amico a et al. clinical/genetic features and natural history in a large cohort of Italian patients. Orphanet J Rare Dis. 2017;12:90,

13. Gupta N, Gothwal S, Satpathy AK, Missaglia S, Tavian D, Das P, et al. Chanarin Dorfman syndrome. A case report with novel nonsense mutation. Gene. 2016;10:359-62.
Ready to submit your research? Choose BMC and benefit from:

- fast, convenient online submission

- thorough peer review by experienced researchers in your field

- rapid publication on acceptance

- support for research data, including large and complex data types

- gold Open Access which fosters wider collaboration and increased citations

- maximum visibility for your research: over $100 \mathrm{M}$ website views per year

At BMC, research is always in progress.

Learn more biomedcentral.com/submissions 